\title{
A Novel SLM Scheme for PAPR Reduction in OFDM Systems
}

\author{
Tian-Ming Ma, Yu-Song Shi, and Ying-Guan Wang \\ Key Laboratory of Wireless Sensor Network and Communication, Shanghai Institute of Microsystem and Information Technology, \\ Chinese Academy of Sciences, No. 778 Chang-Ning Road, Shanghai 200050, China
}

Correspondence should be addressed to Tian-Ming Ma, xdsjtxw@163.com

Received 2 December 2010; Revised 21 February 2011; Accepted 31 March 2011

Academic Editor: W. Hamouda

Copyright () 2011 Tian-Ming Ma et al. This is an open access article distributed under the Creative Commons Attribution License, which permits unrestricted use, distribution, and reproduction in any medium, provided the original work is properly cited.

\begin{abstract}
Orthogonal frequency-division multiplexing (OFDM) is an attractive transmission technique for high-bit-rate communication systems. One major drawback of OFDM is the high peak-to-average power ratio (PAPR) of the transmitter's output signal. A novel selected mapping (SLM) scheme is proposed, which employs matrix transformation, cyclically shifting, and linear combining algorithm to generate new candidates. The novel scheme requires only one IFFT and gets more candidate transmission signals throughout the entire process. The complexity analysis and simulation results show that this algorithm can dramatically reduce computational complexity comparing with the conventional SLM scheme as in Hill et al., 2000; Yang et al., 2009; Wang and Ouyang, 2005; Li et al., 2010; and Heo et al., 2007 under the similar PAPR reduction performance.
\end{abstract}

\section{Introduction}

Orthogonal frequency division multiplexing is an attractive technique for wireless high-rate data transmission due to the minimizing effects over frequency-selective fading channels [1]. As such, OFDM has been chosen for European digital video broadcasting (DVB), wireless local area networks standards (WLANs) (802.11), and digital audio broadcasting (DAB) and is being considered for the long-term evolution of 3GPP.

However, compared with signal carrier signals, OFDM inherits some drawbacks such as sensitivity to synchronization errors and high PAPR at the transmitter, because an OFDM signal is the sum of many narrowband signals in the time domain [2]. The high PAPR can cause the signal distortion such as the in-band distortion and the outof-band radiation due to the nonlinearity of high-power amplifier (HPA) and induces the degradation of bit error rate (BER). Thus, it is highly desirable to reduce the PAPR of an OFDM signal.

Several schemes have been proposed for reducing the PAPR of OFDM signals over the last decade. The PAPR schemes can be classified according to whether they are deterministic or probabilistic. Deterministic schemes, such as clipping [3], deliberately clip the peak amplitude of the OFDM signal to some desired maximum level. Probabilistic schemes, such as partial transmit sequences (PTS) [4, 5] and SLM [6-12], statistically improve the characteristics of the PAPR distribution of the OFDM signals without signal distortion.

It is well known that conventional SLM scheme is a promising PAPR reduction technique for OFDM systems, but it has its own weak points. In this paper, a novel SLM scheme is proposed to overcome its shortcomings. This paper is organized as follows: Section 2 introduces the structure of the conventional SLM scheme. In Section 3, a novel PAPR reduction scheme is proposed, and its principle is given. Section 4 presents a comparison of the novel scheme and the conventional scheme, the scheme in [5, 9-12] in terms of PAPR reduction performances, and their computational complexity, and finally, the brief conclusions are given in Section 5.

\section{Principles of the SLM Scheme}

In the continuous time domain, an OFDM signal $x_{t}$ of $N$ carriers can be expressed as

$$
x_{t}=\frac{1}{\sqrt{N}} \sum_{n=0}^{N-1} X_{n} e^{j 2 \pi n \Delta f t}, \quad 0 \leq t \leq T,
$$


where $X_{n}$ is the data symbol of the $n$th subcarrier, $N$ is the number of subcarriers, $\Delta f$ is the frequency between subcarriers, and $T=1 / \Delta f$ is the OFDM symbol duration. This OFDM signal can be expressed by the vector form, $\mathbf{x}=\left[x_{0}, x_{1}, \ldots, x_{N-1}\right]^{T}$ which is corresponds to the IFFT of $\mathbf{X}=\left[X_{0}, X_{1}, \ldots, X_{N-1}\right]^{T}$.

The PAPR of OFDM signal sequence $\mathbf{x}$ is defined as the ratio between the maximum instantaneous power and its average power, which can be written as

$$
\operatorname{PAPR}(\mathbf{x}) \triangleq \frac{\max _{0 \leq t \leq N-1}\left|x_{t}\right|^{2}}{\mathrm{E}\left[\left|x_{t}\right|^{2}\right]},
$$

where $\mathrm{E}[\cdot]$ denotes the expectation operator.

In the conventional SLM scheme, $K$ alternative input symbol sequences $\mathbf{S}^{(i)}, 1 \leq i \leq K$, are generated by the component-wise vector multiplication of the input symbol sequence $\mathbf{X}$ and $K$ phase sequences $\boldsymbol{\gamma}^{(i)}=$ $\left[b_{0}^{(i)}, b_{1}^{(i)}, \ldots, b_{N-1}^{(i)}\right]^{T}, 1 \leq i \leq K$; that is,

$$
\begin{aligned}
\mathbf{S}^{(i)} & =\left[S_{0}^{(i)}, S_{1}^{(i)}, \ldots, S_{N-1}^{(i)}\right]^{T} \\
& =\mathbf{X} \otimes \boldsymbol{\gamma}^{(i)} \\
& =\left[X_{0} b_{0}^{(i)}, X_{1} b_{1}^{(i)}, \ldots, X_{N-2} b_{N-2}^{(i)}, X_{N-1} b_{N-1}^{(i)}\right]^{T}, \\
& 1 \leq i \leq K,
\end{aligned}
$$

where $\otimes$ denotes the component-wise multiplication of two vectors. $b_{\mu}^{(i)}=\exp \left(j \varphi_{\mu}^{(i)}\right)$ is the rotation factor, $n=0, \ldots$, $N-1 . \varphi_{\mu}^{(i)}$ is homogeneous distributed in $[0,2 \pi)$. To simplify the array multiplication in (3), we let $\gamma^{(1)}=[1,1, \ldots, 1]^{T}$ and choose $b_{k}^{(i)}, 2 \leq i \leq K, k=0,1, \ldots, N-1$, in vector $\gamma^{(i)}$ from the set $\{ \pm 1, \pm j\}$. Then, all $K$ frames are transformed into the time domain using the IFFT, and the one with the lowest PAPR is selected for transmission.

Just as other PAPR reduction schemes, conventional SLM also has its disadvantages: firstly, the selected signal index, called side information (SI), must also be transmitted to allow for the recovery of the original data block at the receiver side, which will inevitably lead to a decrease in data rate. Secondly, in order to improve the PAPR reduction performance of SLM scheme, we have to increase the number of phase sequences. However, the computational complexity of SLM scheme linearly increases as the number of phase sequences increases, which corresponds to the number of IFFTs required to generate the alternative OFDM signals.

\section{A Novel SLM Scheme}

Over the last decade, various methods have been used to improve the drawbacks of conventional SLM technique. Some scholars have invented blind SLM algorithms so that no side information (SI) needs to be sent $[6,7]$. But more concerning improving direction is focused on reducing the computational complexity of SLM scheme. Wang and Li have invented a new scheme to reduce PAPR [8], its applications, however, are only suitable for space-frequency block coding (SFBC) MIMO-OFDM system. Yang et al. have introduced a method of combining the originally time domain sequence linearly with its cyclically shifted sequences to generate the new candidates [9], but only achieved poorer PAPR reduction performance. Hill et al. have combined cyclic shifts of the IFFT subblock output with PTSs to improve the PAPR reduction performance [5]. Wang and Ouyang have proposed to use a low-complexity method to replace IFFTs in the SLM scheme [10], even using random phase rotation vector and cyclically shifting to enhance the PAPR reduction performance [11], which gives us a good idea to further improve its algorithm.

In this section, a novel SLM scheme is proposed which is based on the matrix transformation in [10]. Unlike the shifting algorithm in [11], our scheme uses cyclically shifting to replace the fixed coefficient linear addition in [12] and further improves this algorithm to generate more sequences. Moreover, this algorithm is also different from PTS/CSS in [5], which cyclically shifting the data signal instead of increasing the phase rotations after they were phase rotated to reduce PAPR. The most practicability of our scheme is that it has lower computational complexity than the conventional scheme, the schemes in [5, 9-12] under the similar PAPR reduction performance, which is shown and discussed in Section 4.

According to [10], suppose that $\mathbf{s}^{(1)}$ is the IFFT output signal $\mathbf{s}^{(i)}, i=1$, corresponding to the frequency-domain signals

$$
\mathbf{s}^{(1)}=\operatorname{IFFT}\left[\mathbf{S}^{(1)}\right]=\mathbf{Q} \mathbf{S}^{(1)}=\mathbf{Q X},
$$

where $\mathbf{Q}$ is the IFFT matrix given by

$$
\mathbf{Q}=\frac{1}{N}\left(\begin{array}{ccccc}
1 & 1 & 1 & \cdots & 1 \\
1 & W_{N}^{-1} & W_{N}^{-2} & \cdots & W_{N}^{-(N-1)} \\
1 & W_{N}^{-2} & W_{N}^{-4} & \cdots & W_{N}^{-2(N-1)} \\
\vdots & \vdots & \vdots & \ddots & \vdots \\
1 & W_{N}^{-(N-1)} & W_{N}^{-2(N-1)} & \cdots & W_{N}^{-(N-1)(N-1)}
\end{array}\right) \text {, }
$$

with $W_{N}=e^{-j 2 \pi / N}$.

From (4), we can easily obtain

$$
\mathbf{X}=\mathbf{Q}^{-1} \mathbf{s}^{(1)},
$$

where $\mathbf{Q}^{-1}$ denotes the inverse of $\mathbf{Q}$.

According to the matrix theory, (3) can be re-expressed as

$$
\mathbf{S}^{(i)}=\mathbf{R}_{i} \mathbf{X}
$$

where

$$
\mathbf{R}_{i}=\left(\begin{array}{cccc}
b_{0}^{(i)} & & & 0 \\
& b_{1}^{(i)} & & \\
& & \ddots & \\
0 & & & b_{N-1}^{(i)}
\end{array}\right)
$$




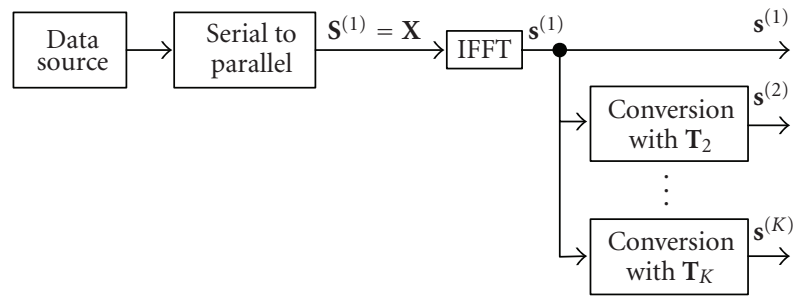

FIgure 1: Idea of conversion with $\mathbf{T}_{r}$ for IFFT computation.

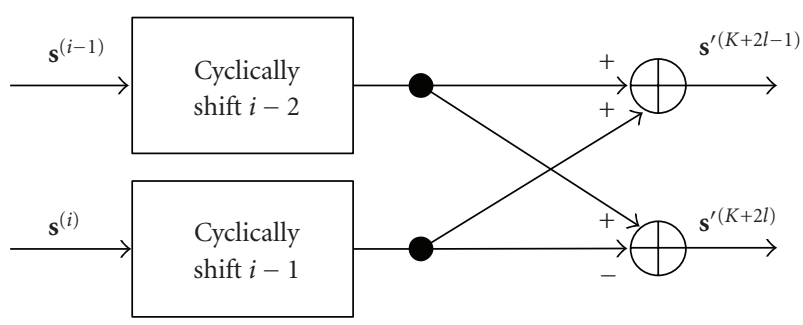

FIGURE 2: Algorithm of cyclically shifting for generating new sequences.

is referred to as the phase rotation matrix corresponding to the phase sequences $\gamma^{(i)}$.

Let $\mathbf{s}^{(t)}$ be a IFFT output signal $\mathbf{s}^{(i)}, 2 \leq i \leq K$, then we can get

$$
\mathbf{s}^{(t)}=\operatorname{IFFT}\left[\mathbf{S}^{(t)}\right]=\mathbf{Q} \mathbf{S}^{(t)}=\mathbf{Q R}_{t} \mathbf{X}
$$

From (6), we can rewrite (9)

$$
\mathbf{s}^{(t)}=\mathbf{T}_{t} \mathbf{s}^{(1)},
$$

where $\mathbf{T}_{t}=\mathbf{Q R}_{t} \mathbf{Q}^{-1}$ is a conversion matrix.

Then, all the IFFT output signals $\mathbf{s}^{(i)}, 1 \leq i \leq K$, can be expressed by (4) and (10), as shown in Figure 1.

In order to achieve large PAPR reduction, a large number of candidate signal sequences should be required, but it will increase computational complexity, because each alternative OFDM signal sequence should be generated by (10). In [12], $K(K-1)$ additional alternative OFDM signal sequences from $K$ IFFTed alternative signal sequences without performing IFFT are generated by linear combination, and $c_{m}$ and $c_{n}$ are two fixed coefficient $1 / \sqrt{2}$ and $\pm(1 / \sqrt{2}) j$. To generate these sequences, we should use $2 N(K-1)$ multiplications and $N\left(K^{2}-K\right)$ additions, where $N$ is the number of subcarriers and $K$ is the number of IFFTed alternative signal sequences.

By simulation experiments, however, we find that if we cyclically shift $K$ IFFTed sequences regularly and linear combine them to generate $K(K-1)$ additional sequences, the PAPR performance of the SLM scheme will generally be the same as even slightly better than that of in [12], which can be seen in Figure 9. Furthermore, the number of complex multiplications and complex additions is $N(K-1)$ and $N\left(K^{2}-K\right)$, respectively. The algorithm of cyclically shifting and linear combination is described in Figure 2.

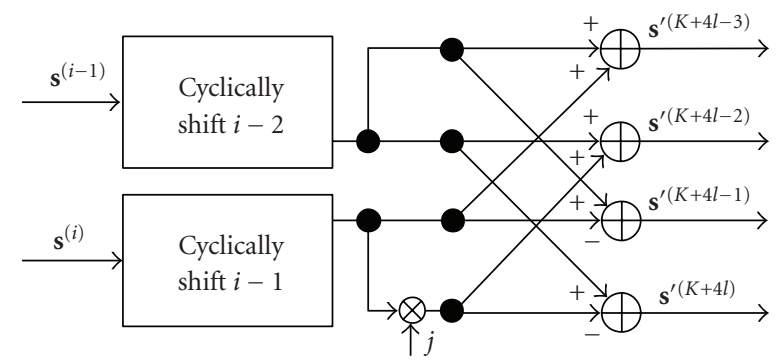

FIGURE 3: Algorithm of improved cyclically shifting for generate new sequences.

Let $\mathbf{s}^{(i-1)}$ and $\mathbf{s}^{(i)}$ be the two arbitrary $K$ IFFTed alternative signal sequences, the linear combination of these two sequence can be given as

$$
\begin{aligned}
\mathbf{s}^{\prime(K+2 l-1)} & =\operatorname{circular}\left(\mathbf{s}^{(i-1)}, i-2\right)+\operatorname{circular}\left(\mathbf{s}^{(i)}, i-1\right), \\
\mathbf{s}^{\prime(K+2 l)} & =\operatorname{circular}\left(\mathbf{s}^{(i-1)}, i-2\right)-\operatorname{circular}\left(\mathbf{s}^{(i)}, i-1\right),
\end{aligned}
$$

where circular( $\left.\mathbf{s}^{(i-1)}, i-2\right)$ denotes right cyclically shifting $\mathbf{s}^{(i-1)}$ by $i-2$, which can be expressed as

$$
\begin{aligned}
& \operatorname{circular}\left(\mathbf{s}^{(i-1)}, i-2\right) \\
& \quad=\left[\mathbf{s}_{i-2}^{(i-1)}, \mathbf{s}_{i-1}^{(i-1)}, \ldots, \mathbf{s}_{N-1}^{(i-1)}, \mathbf{s}_{0}^{(i-1)}, \mathbf{s}_{1}^{(i-1)}, \ldots, \mathbf{s}_{i-4}^{(i-1)}, \mathbf{s}_{i-3}^{(i-1)}\right]^{T} \\
& =\mathbf{E}_{i-2} \cdot \mathbf{s}^{(i-1)},
\end{aligned}
$$

where

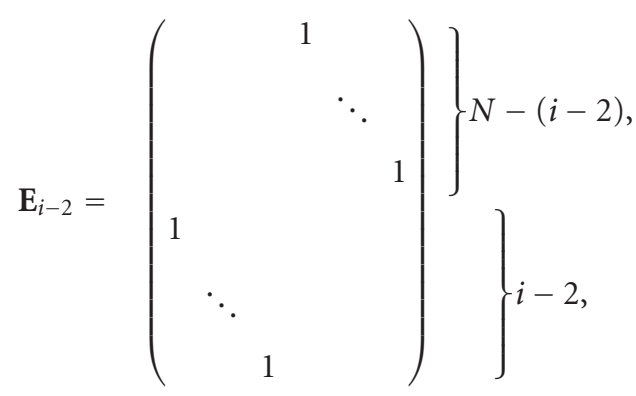

and $\operatorname{circular}\left(\mathbf{s}^{(i)}, i-1\right)$ can be done in the same manner.

In addition, we can improve this algorithm to increase the number of signal sequences, as shown in Figure 3. 


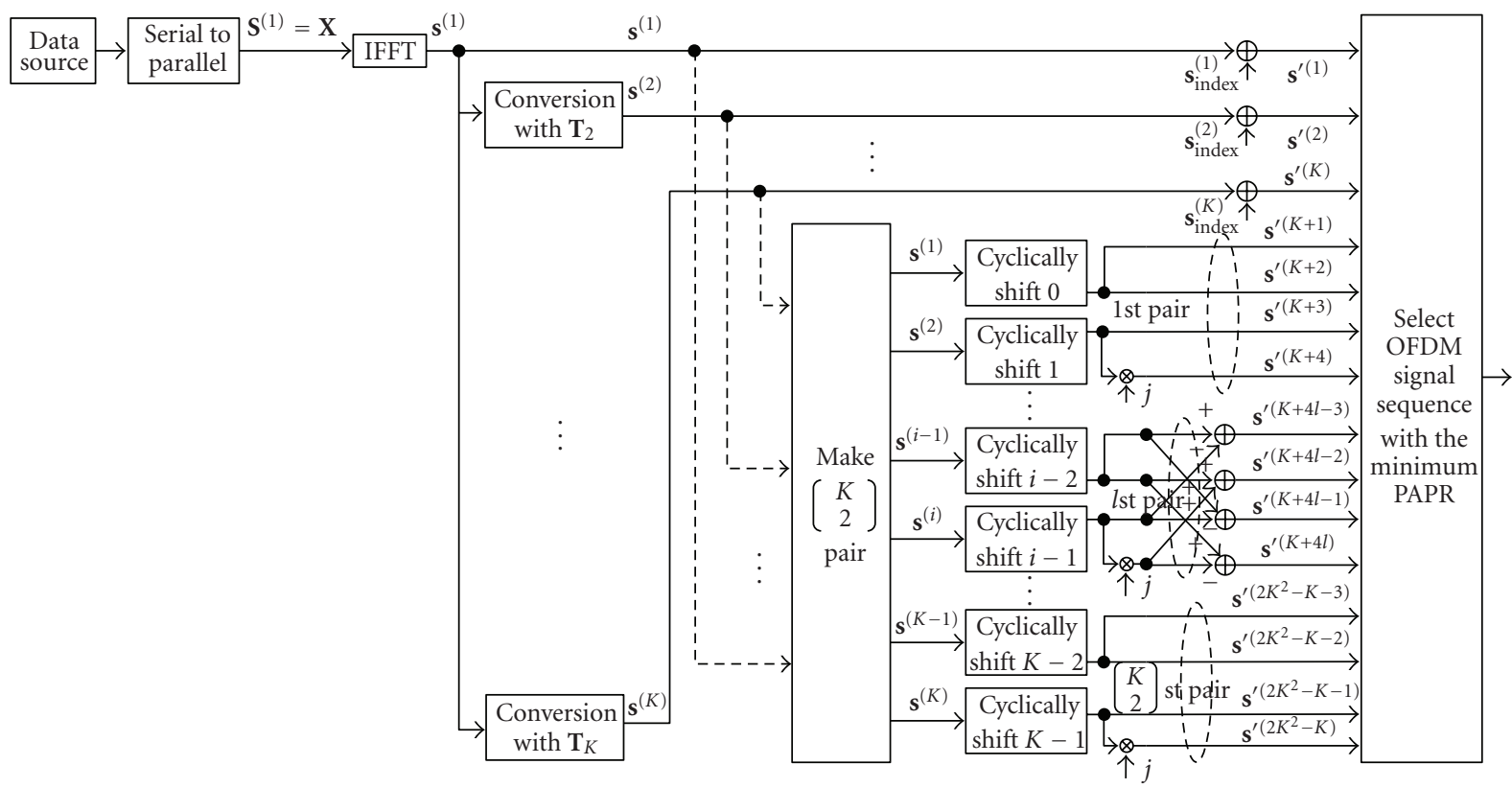

FIgURE 4: Block diagram of the novel SLM scheme.

The improved linear combination of these two sequences can be given as

$$
\begin{aligned}
\mathbf{s}^{\prime(K+4 l-3)} & =\operatorname{circular}\left(\mathbf{s}^{(i-1)}, i-2\right)+\operatorname{circular}\left(\mathbf{s}^{(i)}, i-1\right) \\
& =\operatorname{IFFT}\left[\mathbf{E}_{i-2} \mathbf{S}^{(i-1)}+\mathbf{E}_{i-1} \mathbf{S}^{(i)}\right] \\
\mathbf{s}^{\prime(K+4 l-2)} & =\operatorname{circular}\left(\mathbf{s}^{(i-1)}, i-2\right)+j \cdot \operatorname{circular}\left(\mathbf{s}^{(i)}, i-1\right) \\
& =\operatorname{IFFT}\left[\mathbf{E}_{i-2} \mathbf{S}^{(i-1)}+j \mathbf{E}_{i-1} \mathbf{S}^{(i)}\right], \\
\mathbf{s}^{\prime(K+4 l-1)} & =\operatorname{circular}\left(\mathbf{s}^{(i-1)}, i-2\right)-\operatorname{circular}\left(\mathbf{s}^{(i)}, i-1\right) \\
& =\operatorname{IFFT}\left[\mathbf{E}_{i-2} \mathbf{S}^{(i-1)}-\mathbf{E}_{i-1} \mathbf{S}^{(i)}\right], \\
\mathbf{s}^{\prime(K+4 l)} & =\operatorname{circular}\left(\mathbf{s}^{(i-1)}, i-2\right)-j \cdot \operatorname{circular}\left(\mathbf{s}^{(i)}, i-1\right) \\
& =\operatorname{IFFT}\left[\mathbf{E}_{i-2} \mathbf{S}^{(i-1)}-j \mathbf{E}_{i-1} \mathbf{S}^{(i)}\right],
\end{aligned}
$$

thus, $4 \times\left(\begin{array}{l}K \\ 2\end{array}\right)=2 K^{2}-2 K$ additional alternative OFDM signal sequences can be generated from cyclically shifting and linear combining algorithm without new IFFT operations.

Furthermore, the information of the phase sequence used for the transmitted signal must be conveyed to the receiver in the SLM scheme. In the novel SLM scheme, the input symbol sequence $\mathbf{X}$ consist of $\mathbf{X}_{\text {data }}$ and $\mathbf{X}_{\text {index }}$ which are the data symbol sequence and the index symbol sequence. The index signal is represented as $\mathbf{x}_{\text {index }}=\operatorname{IFFT}\left(\mathbf{X}_{\text {index }}\right), 1 \leq i \leq 4$ and is added after IFFT of $\mathbf{X}_{\text {index }}$.
Finally, the number of total $2 K^{2}-K$ signal sequences can be written as

$$
\begin{aligned}
& \{\underbrace{\underbrace{\mathbf{s}^{(1)}, \mathbf{s}^{(2)}, \ldots, \mathbf{s}^{(K)}},}_{K} \\
& \underbrace{\mathbf{s}^{(1)}+b \mathbf{E}_{1} \mathbf{s}^{(2)}, \mathbf{s}^{(1)}+b \mathbf{E}_{2} \mathbf{s}^{(3)}, \ldots, \mathbf{E}_{K-2} \mathbf{s}^{(K-1)}+b \mathbf{E}_{K-1} \mathbf{s}^{(K)}}_{2\left(K^{2}-K\right)}\},
\end{aligned}
$$

where $b \in\{1, j,-1,-j\}$, for $i=1$,

$$
\begin{aligned}
\mathbf{s}^{(1)} & =\operatorname{IFFT}\left[\mathbf{S}_{\text {data }}^{(1)}\right]+\operatorname{IFFT}\left[\mathbf{S}_{\text {index }}^{(1)}\right] \\
& =\mathbf{Q X}_{\text {data }}+\mathbf{Q} \mathbf{X}_{\text {index }},
\end{aligned}
$$

for $2 \leq i \leq K$,

$$
\begin{aligned}
\mathbf{s}^{(i)} & =\operatorname{IFFT}\left[\mathbf{S}_{\text {data }}^{(i)}\right]+\operatorname{IFFT}\left[\mathbf{S}_{\text {index }}^{(i)}\right] \\
& =\mathbf{Q R}_{i} \mathbf{X}_{\text {data }}+\mathbf{Q} \mathbf{R}_{i} \mathbf{X}_{\text {index }},
\end{aligned}
$$

and for $K+1 \leq i \leq 2 K^{2}-K$ and $1 \leq m<n \leq K$,

$$
\begin{aligned}
\mathbf{s}^{(i)} & =\operatorname{IFFT}\left[\mathbf{E}_{m-1} \mathbf{S}_{\text {data }}^{(m)}+b \mathbf{E}_{n-1} \mathbf{S}_{\text {data }}^{(n)}\right]+\operatorname{IFFT}\left[\mathbf{S}_{\text {index }}^{(i)}\right] \\
& =\left(\mathbf{E}_{m-1} \mathbf{Q R}_{m}+b \mathbf{E}_{n-1} \mathbf{Q R}_{n}\right) \mathbf{X}_{\text {data }}+\mathbf{Q R}_{i} \mathbf{X}_{\text {index }} .
\end{aligned}
$$

The final novel SLM scheme is described in Figure 4.

\section{Performance Results}

The complexity analysis and simulation results demonstrate the effectiveness of the proposed scheme. 


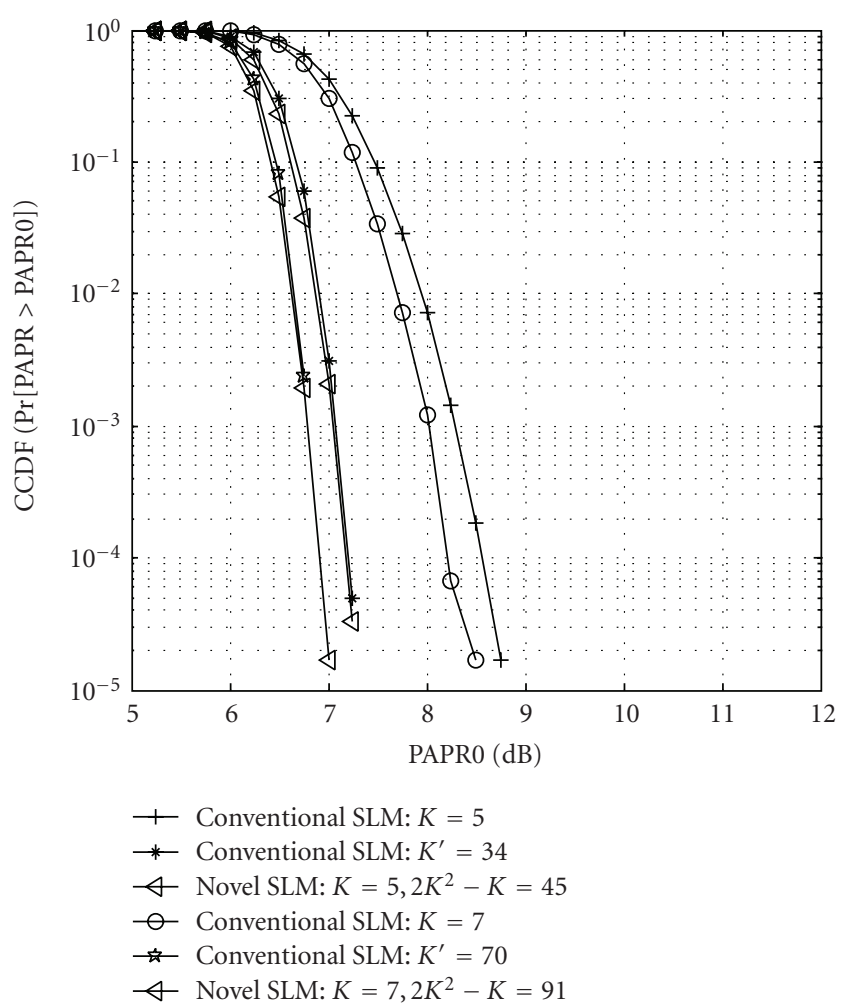

FIGURE 5: PAPR reduction performance of the novel SLM scheme and the conventional SLM scheme.

TABLE 1: Simulation parameters.

\begin{tabular}{lc}
\hline Modulation and demodulation & QPSK \\
Allocated bandwidth & $8 \mathrm{MHz}$ \\
Channel model & i.i.d. quasi-static \\
Rayleigh fading \\
Coding rate & $1 / 2$ \\
Number of random blocks & 60,000 \\
Number of subcarriers $(N)$ & 256 \\
Number of signal sequences $(K)$ & 5,7 , and \\
\end{tabular}

4.1. Simulation Results. PAPR reduction performances of the conventional and novel SLM schemes were investigated by MATLAB simulations. The basic system parameters for the simulations are summarized in Table 1.

Figure 5 shows a comparison of PAPR reduction performance for the novel SLM scheme with $K=5,7$ and the conventional one with $K=5,7, K^{\prime}=34,70$, respectively. When $K=5$, the PAPR of the conventional SLM scheme is about $8.6 \mathrm{~dB}$ at $\mathrm{CCDF}=0.1 \%$, the definition of CCDF is given as complementary cumulative distribution function. With the increase of the candidates, both the conventional scheme and the novel one can continue to improve the PAPR. The result shows that the novel scheme when $K=5,7$ has almost the same performance as that of in conventional one when $K^{\prime}=34,70$, respectively. That is to say, the performance of novel SLM with $\left(2 K^{2}-K\right)$ alternative signal

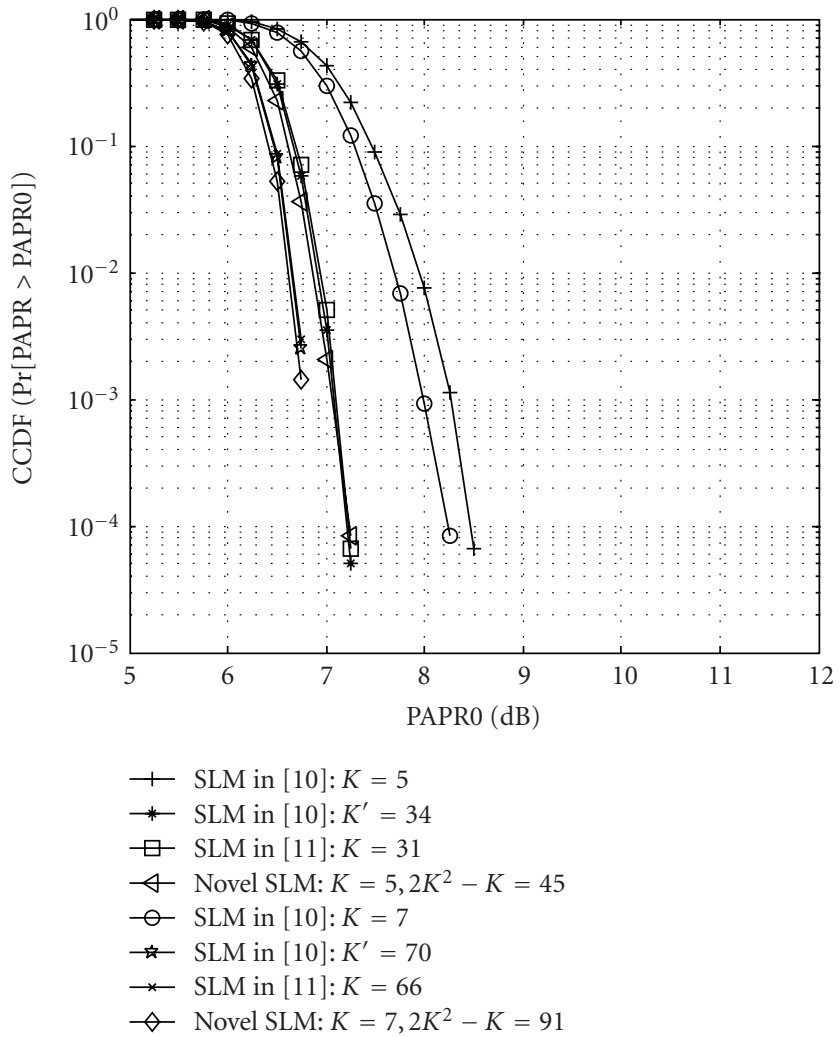

FIGURE 6: PAPR reduction performance of the novel SLM scheme and the SLM schemes in $[10,11]$.

sequences is generally equal to that of in conventional one with $K^{\prime}=\left\lfloor 0.77\left(2 K^{2}-K\right)\right\rfloor$ alternative signal sequences, where $\lfloor\cdot\rfloor$ represents the integer part of the $0.77\left(2 K^{2}-K\right)$.

Figure 6 depicts a performance comparison in PAPR reduction of the novel scheme with $K=5,7$, the scheme in [10] with $K=5,7, K^{\prime}=34,70$ and the scheme in [11] with $K=31,66$, respectively. From Figure 6 we can find that the performance of conventional scheme is almost the same as that of scheme in [10]. We can also know that in order to get the similar PAPR reduction performance, the schemes in [10] need more alternative signal sequences, which is consistent with the conclusion in [11].

In Figure 7, the proposed scheme with $K=5,7$ has almost the same performance compared with the SLM scheme in [9] with $K=38,75$, respectively. From inspection, it is easy to draw a conclusion that cyclic shifts can reduce the complexity at the cost of mitigating the PAPR reduction performance when comparing with the conventional algorithm.

Figure 8 demonstrates PAPR performance of the novel SLM scheme with $K=5,7$ and the PTS/CSS scheme in [5] with $W=4, S=2, V=3,5$, respectively, where $W$ is phase factor, $V$ is the number of subblocks and $S$ is the number of cyclic shifting options. It is worthy of note that when CCDF $=10^{-3}$, the performance of the scheme in [5] is worse than that of the proposed scheme by about $1 \mathrm{~dB}$; that is, the novel SLM scheme can achieve much better reduction performance comparing with the scheme in [5]. 
TABLE 2: Computational complexity of the conventional SLM and the novel SLM schemes when $N=256$ and 1024 .

\begin{tabular}{|c|c|c|c|c|c|c|}
\hline & $\begin{array}{l}\text { Conventional } \\
\text { SLM, } K=34\end{array}$ & $\begin{array}{l}\text { Novel } \\
\text { SLM, } \\
K=5\end{array}$ & CCRR & $\begin{array}{l}\text { Conventional } \\
\text { SLM, } K=70\end{array}$ & $\begin{array}{l}\text { Novel } \\
\text { SLM, } \\
K=7 \\
\end{array}$ & CCRR \\
\hline \multirow[t]{2}{*}{ IFFTs } & 34 & 5 & & 70 & 7 & \\
\hline & \multicolumn{6}{|c|}{$N=256$} \\
\hline Complex multiplications & 34,816 & 2,048 & $94.1 \%$ & 71,680 & 2,560 & $96.4 \%$ \\
\hline \multirow[t]{2}{*}{ Complex additions } & 69,632 & 15,360 & $77.9 \%$ & 143,360 & 28,160 & $80.4 \%$ \\
\hline & \multicolumn{6}{|c|}{$N=1024$} \\
\hline Complex multiplications & 174,080 & 9,216 & $94.7 \%$ & 358,400 & 11,264 & $96.9 \%$ \\
\hline Complex additions & 348,160 & 63,488 & $81.8 \%$ & 716,800 & 114,688 & $84.0 \%$ \\
\hline
\end{tabular}

TABLE 3: Computational complexity of the SLM schemes in [10] and the novel SLM schemes when $N=256$ and 1024 .

\begin{tabular}{|c|c|c|c|c|c|c|}
\hline & $\begin{array}{c}\text { SLM in } \\
{[10] \text {, }} \\
K=34\end{array}$ & $\begin{array}{l}\text { Novel } \\
\text { SLM, } \\
K=5\end{array}$ & CCRR & $\begin{array}{c}\text { SLM in } \\
{[10],} \\
K=70\end{array}$ & $\begin{array}{c}\text { Novel } \\
\text { SLM, } \\
K=7\end{array}$ & CCRR \\
\hline \multirow[t]{2}{*}{ IFFTs } & 1 & 5 & & 1 & 7 & \\
\hline & \multicolumn{6}{|c|}{$N=256$} \\
\hline Complex multiplications & 1,024 & 2,048 & $-100 \%$ & 1,024 & 2,560 & $-150 \%$ \\
\hline \multirow[t]{2}{*}{ Complex additions } & 27,392 & 15,360 & $43.9 \%$ & 55,040 & 28,160 & $48.8 \%$ \\
\hline & \multicolumn{6}{|c|}{$N=1024$} \\
\hline Complex multiplications & 5,120 & 9,216 & $-80 \%$ & 5,120 & 11,264 & $-120 \%$ \\
\hline Complex additions & 111,616 & 63,488 & $43.1 \%$ & 222,208 & 114,688 & $48.4 \%$ \\
\hline
\end{tabular}

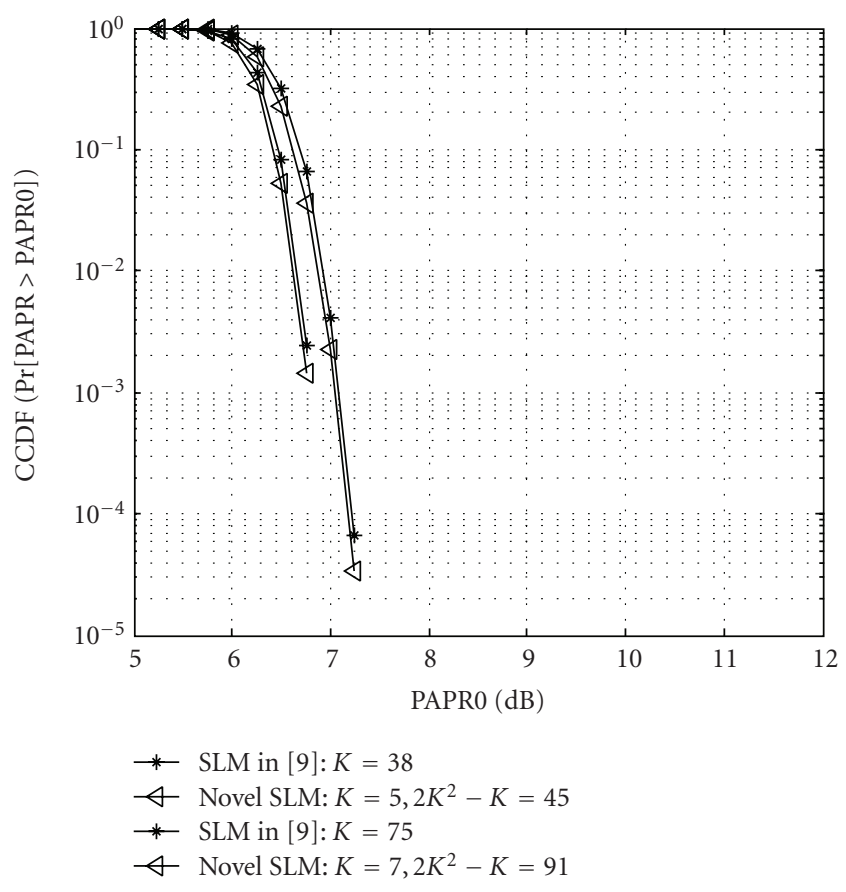

FIGURE 7: PAPR reduction performance of the novel SLM scheme and the SLM scheme in [9].

Figure 9 gives us a comparison in PAPR performance of the novel scheme, the scheme in Figure 2, and the scheme in [12]. It can be seen that the algorithm in Figure 2 not only have lower computation complexity but also have

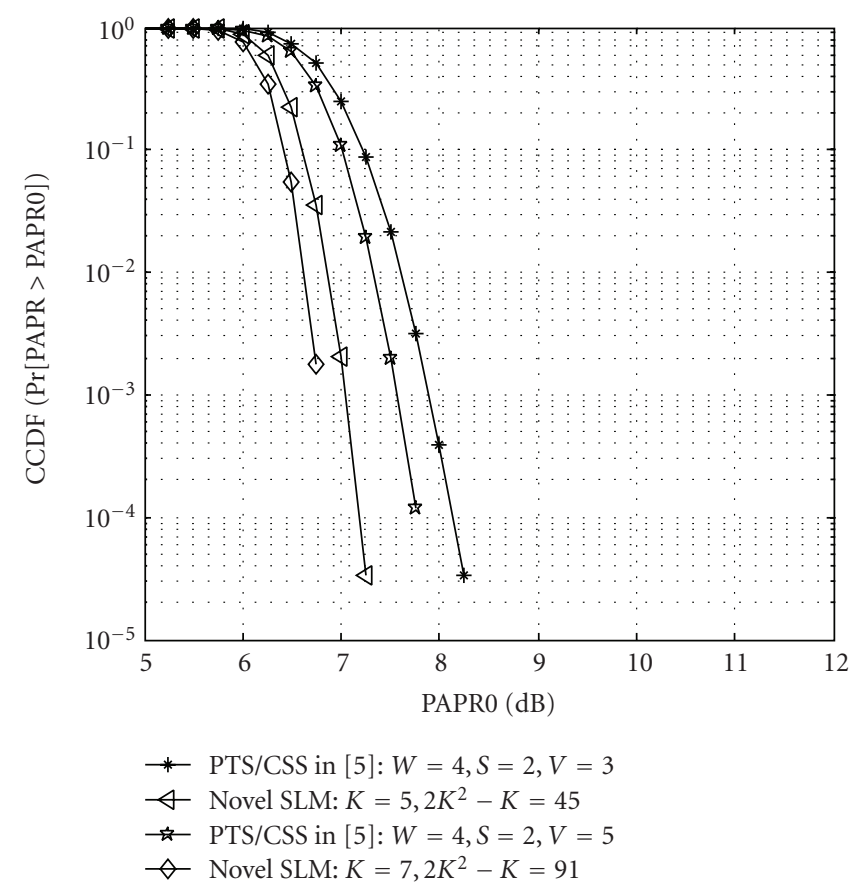

FIGURE 8: PAPR reduction performance of the novel SLM scheme and the PTS scheme in [5].

slightly better PAPR reduction than the algorithm in [12] whenever $K=5$ or 7 , which shows the superiority of the algorithm. Another useful conclusion is that when $K=7$, the PAPR performance of scheme in [12] has almost the same 
TABLE 4: Computational complexity of the SLM schemes in [11] and the novel SLM schemes when $N=256$ and 1024 .

\begin{tabular}{|c|c|c|c|c|c|c|}
\hline & $\begin{array}{c}\text { SLM in } \\
{[11],} \\
K=31\end{array}$ & $\begin{array}{l}\text { Novel } \\
\text { SLM, } \\
K=5\end{array}$ & CCRR & $\begin{array}{c}\text { SLM in } \\
{[11],} \\
K=66\end{array}$ & $\begin{array}{c}\text { Novel } \\
\text { SLM, } \\
K=7\end{array}$ & CCRR \\
\hline IFFTs & 1 & 5 & & 1 & 7 & \\
\hline & \multicolumn{6}{|c|}{$N=256$} \\
\hline Complex multiplications & 1,024 & 2,048 & $-100 \%$ & 1,024 & 2,560 & $-150 \%$ \\
\hline \multirow[t]{2}{*}{ Complex additions } & 25,856 & 15,360 & $40.6 \%$ & 52,736 & 28,160 & $46.6 \%$ \\
\hline & \multicolumn{6}{|c|}{$N=1024$} \\
\hline Complex multiplications & 5,120 & 9,216 & $-80 \%$ & 5,120 & 11,264 & $-120 \%$ \\
\hline Complex additions & 105,472 & 63,488 & $39.8 \%$ & 212,992 & 114,688 & $46.2 \%$ \\
\hline
\end{tabular}

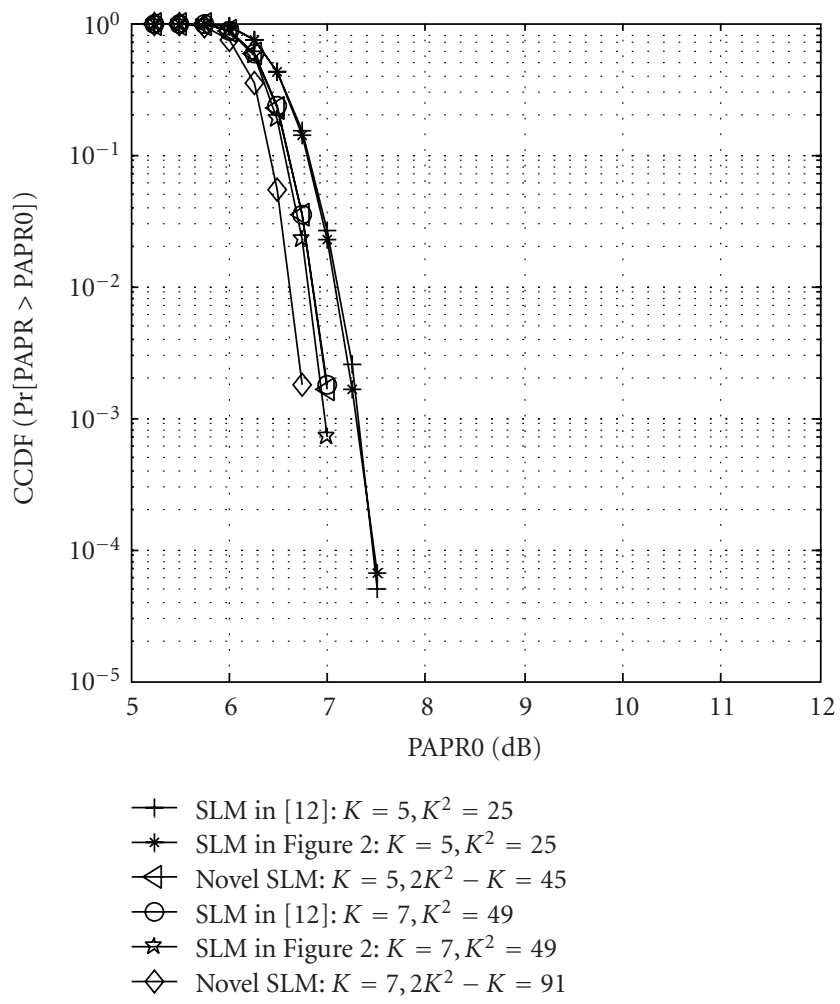

FIGURE 9: PAPR reduction performance of the novel SLM scheme, SLM scheme in Figure 3, and the SLM scheme in [12].

performance of novel scheme with $K=5$. In particular, the performance of novel SLM with $\left(2 K^{2}-K\right)$ alternative signal sequences is generally equal to that of in [12] with $K^{\prime}=\left\lfloor 0.92\left(2 K^{2}-K\right)\right\rfloor$ alternative signal sequences.

4.2. Computational Complexity. The computational complexity reduction ratio (CCRR) of the novel SLM scheme over the conventional SLM scheme is defined as

$$
\begin{aligned}
\text { CCRR }=\left(1-\frac{\text { complexity of the proposed scheme }}{\text { complexity of the original scheme }}\right) \\
\\
\times 100(\%)
\end{aligned}
$$

It is well known that an $N$-point IFFT requires $(N / 2) \log _{2} N$ multiplications and $N \log _{2} N$ additions. Thus, the conventional SLM scheme with $K$ individual phase sequences requires $K$ IFFT operations; therefore, the total numbers of multiplications and additions are $(K N / 2) \log _{2} N$ and $K N \log _{2} N$, respectively. While for the novel SLM scheme, using cyclically shifting and linear combining algorithm to add $2\left(K^{2}-K\right)$ sequences will take $(K-1) N$ multiplications and $2\left(K^{2}-K\right) N$ additions. Therefore, the total of computational complexity is $(N / 2) \log _{2} N+(K-1) N$ complex multiplications and $N \log _{2} N+\left(2 K^{2}+K-3\right) N$ complex additions. The CCRR of the novel schemes over the conventional schemes with typical values of $K$ and $N$ is given in Table 2.

From [10], we know that it takes $(N / 2) \log _{2} N$ multiplications and $N \log _{2} N+3(K-1) N$ additions to generate $K$ sequences. Table 3 illustrates the CCRR for both the proposed scheme and SLM schemes in [10].

According to $[11]$, scheme III requires $(N / 2) \log _{2} N$ multiplications and $N \log _{2} \mathrm{~N}+3 \mathrm{KN}$ additions to generate $\mathrm{K}$ sequences. Table 4 shows the CCRR comparison of the novel schemes over the SLM schemes in [11].

As is discussed above, using the method of cyclically shift the signal sequence and combining it with the shifting sequences to generate $K-1$ symbol sequences will take $(N / 2) \log _{2} N+N(K-1)$ multiplications and $N \log _{2} N+$ $N(K-1)$ additions. Therefore, the CCRR of the novel schemes over the SLM schemes in [9] is shown in Table 5.

From [5], we know that PTS/CSS scheme needs $N(V-$ 1) $W^{(V-1)}$ multiplications and $N V(W S)^{(V-1)}$ additions to generate $V$ sequences. We can see that our proposed SLM schemes involve much lower computational complexity than the scheme in [5] whenever $N=256$ or 1024. The CCRR of the novel schemes over the SLM schemes in [5] is shown in Table 6.

According to [12], we know that it takes $2 N(K-1)+$ $(N K / 2) \log _{2} N$ multiplications and $N\left(K^{2}-K\right)+N K \log _{2} N$ additions to generate $K$ sequences. Table 7 shows the CCRR of the novel schemes over the SLM schemes in [12].

From the tables listed above, we can draw a conclusion that the computation complexity of novel SLM scheme is lower than the conventional scheme and the scheme in [5, 12] when they have the similar PAPR reduction performance. Furthermore, the novel scheme becomes computationally 
TABLE 5: Computational complexity of the SLM schemes in [9] and the novel SLM schemes when $N=256$ and 1024.

\begin{tabular}{|c|c|c|c|c|c|c|}
\hline & $\begin{array}{c}\text { SLM in } \\
\text { [9], } \\
K=38\end{array}$ & $\begin{array}{l}\text { Novel } \\
\text { SLM, } \\
K=5\end{array}$ & CCRR & $\begin{array}{c}\text { SLM in } \\
\text { [9], } \\
K=75\end{array}$ & $\begin{array}{l}\text { Novel } \\
\text { SLM, } \\
K=7\end{array}$ & CCRR \\
\hline \multirow[t]{2}{*}{ IFFTs } & 1 & 5 & & 1 & 7 & \\
\hline & \multicolumn{6}{|c|}{$N=256$} \\
\hline Complex multiplications & 10,496 & 2,048 & $80.5 \%$ & 19,968 & 2,560 & $87.2 \%$ \\
\hline \multirow[t]{2}{*}{ Complex additions } & 11,520 & 15,360 & $-33.3 \%$ & 20,992 & 28,160 & $-34.1 \%$ \\
\hline & \multicolumn{6}{|c|}{$N=1024$} \\
\hline Complex multiplications & 43,008 & 9,216 & $78.6 \%$ & 80,896 & 11,264 & $86.1 \%$ \\
\hline Complex additions & 48,128 & 63,488 & $-32.2 \%$ & 86,016 & 114,688 & $-33.3 \%$ \\
\hline
\end{tabular}

TABLE 6: Computational complexity of the SLM schemes in [5] and the novel SLM schemes when $N=256$ and 1024.

\begin{tabular}{|c|c|c|c|c|c|c|}
\hline & $\begin{array}{l}\text { PTS/CSS in } \\
{[5], W=4,} \\
S=2, V=3\end{array}$ & $\begin{array}{c}\text { Novel SLM, } \\
K=5\end{array}$ & CCRR & $\begin{array}{l}\text { PTS/CSS in } \\
{[5], W=4} \\
S=2, V=5\end{array}$ & $\begin{array}{c}\text { Novel SLM, } \\
K=7\end{array}$ & CCRR \\
\hline \multirow[t]{2}{*}{ IFFTs } & 3 & 5 & & 5 & 7 & \\
\hline & \multicolumn{6}{|c|}{$N=256$} \\
\hline Complex multiplications & 8,192 & 2,048 & $75.0 \%$ & 262,144 & 2,560 & $99.0 \%$ \\
\hline \multirow[t]{2}{*}{ Complex additions } & 49,152 & 15,360 & $68.8 \%$ & $5,242,880$ & 28,160 & $99.5 \%$ \\
\hline & \multicolumn{6}{|c|}{$N=1024$} \\
\hline Complex multiplications & 32,768 & 9,216 & $71.9 \%$ & $1,048,576$ & 11,264 & $98.9 \%$ \\
\hline Complex additions & 196,608 & 63,488 & $67.7 \%$ & $20,971,520$ & 114,688 & $99.5 \%$ \\
\hline
\end{tabular}

TABLE 7: Computational complexity of the SLM schemes in [12] and the novel SLM schemes when $N=256$ and 1024 .

\begin{tabular}{|c|c|c|c|}
\hline & SLM in [12], $K=7$ & Novel SLM, $K=5$ & CCRR \\
\hline \multirow[t]{2}{*}{ IFFTs } & 7 & 5 & \\
\hline & \multicolumn{3}{|c|}{$N=256$} \\
\hline Complex multiplications & 10,240 & 2,048 & $80 \%$ \\
\hline \multirow[t]{2}{*}{ Complex additions } & 25,088 & 15,360 & $38.8 \%$ \\
\hline & \multicolumn{3}{|c|}{$N=1024$} \\
\hline Complex multiplications & 48,128 & 9,216 & $80.9 \%$ \\
\hline Complex additions & 114,688 & 63,488 & $44.6 \%$ \\
\hline
\end{tabular}

more efficient as $N$ or $K$ increases. Although the complex multiplications of the scheme in $[10,11]$ and the complex additions of the scheme in [9] are lower than the novel scheme, its total computational complexity, however, is still higher than the latter. These analysis result shows that novel scheme is computationally more efficient than the other schemes. Thus, it is useful for high data rate transmission systems.

\section{Conclusion}

In this paper, a novel SLM scheme is proposed, and its performance is numerically confirmed for the OFDM system proposed in the IEEE 802.16 standard. The results show that as compared with the conventional SLM scheme, the scheme in $[5,9-12]$, using this algorithm can receive significant reduction in the computational complexity keeping the similar PAPR reduction performance. Therefore, it is rather attractive for use in OFDM-based communication systems.

\section{References}

[1] R. Prasad, OFDM for Wireless Communications Systems, Artech House, London, UK, 2004.

[2] S. H. Han and J. H. Lee, "An overview of peak-to-average power ratio reduction techniques for multicarrier transmission," IEEE Wireless Communications, vol. 12, no. 2, pp. 56-65, 2005.

[3] L. Wang and C. Tellambura, "A simplified clipping and filtering technique for PAR reduction in OFDM systems," IEEE Signal Processing Letters, vol. 12, no. 6, pp. 453-456, 2005.

[4] S. S. Kim, M. J. Kim, and T. A. Gulliver, "PAPR reduction of OFDM signals using genetic algorithm PTS technique," IEICE Transactions on Communications, vol. E91-B, no. 4, pp. 11941197, 2008. 
[5] G. R. Hill, M. Faulkner, and J. Singh, "Reducing the peakto-average power ratio in OFDM by cyclically shifting partial transmit sequences," Electronics Letters, vol. 36, no. 6, pp. 560$561,2000$.

[6] I. M. Hussain and I. A. Tasadduq, "PAPR reduction of OFDM signals using autocorrelation based SLM without side information," in Proceedings of the IEEE Canadian Conference on Electrical and Computer Engineering (CCECE '08), pp. 7175, Ontario, Canada, May 2008.

[7] S. Y. Le Goff, S. S. Al-Samahi, and Boon Kien Khoo, "Selected mapping without side information for PAPR reduction in OFDM," IEEE Transactions on Wireless Communications, vol. 8, no. 7, pp. 3320-3325, 2009.

[8] S. H. Wang and C. P. Li, "A low-complexity PAPR reduction scheme for SFBC MIMO-OFDM systems," IEEE Signal Processing Letters, vol. 16, no. 11, pp. 941-944, 2009.

[9] L. Yang, S. Q. Li, K. K. Soo, and Y. M. Siu, "SLM with non-unit magnitude phase factors for PAPR reduction in OFDM," in Proceedings of the International Conference on Communications, Circuits and Systems (ICCCAS '09), pp. 5355, Milpitas, Calif, USA, July 2009.

[10] C. L. Wang and Y. Ouyang, "Low-complexity selected mapping schemes for peak-to-average power ratio reduction in OFDM systems," IEEE Transactions on Signal Processing, vol. 53, no. 12, pp. 4652-4660, 2005.

[11] C. P. Li, S. H. Wang, and C. L. Wang, "Novel low-complexity SLM schemes for PAPR reduction in OFDM systems," IEEE Transactions on Signal Processing, vol. 58, no. 5, pp. 2916-2921, 2010.

[12] S. J. Heo, H. S. Noh, J. S. No, and D. J. Shin, "A modified SLM scheme with low complexity for PAPR reduction of OFDM systems," IEEE Transactions on Broadcasting, vol. 53, no. 4, pp. 804-808, 2007. 

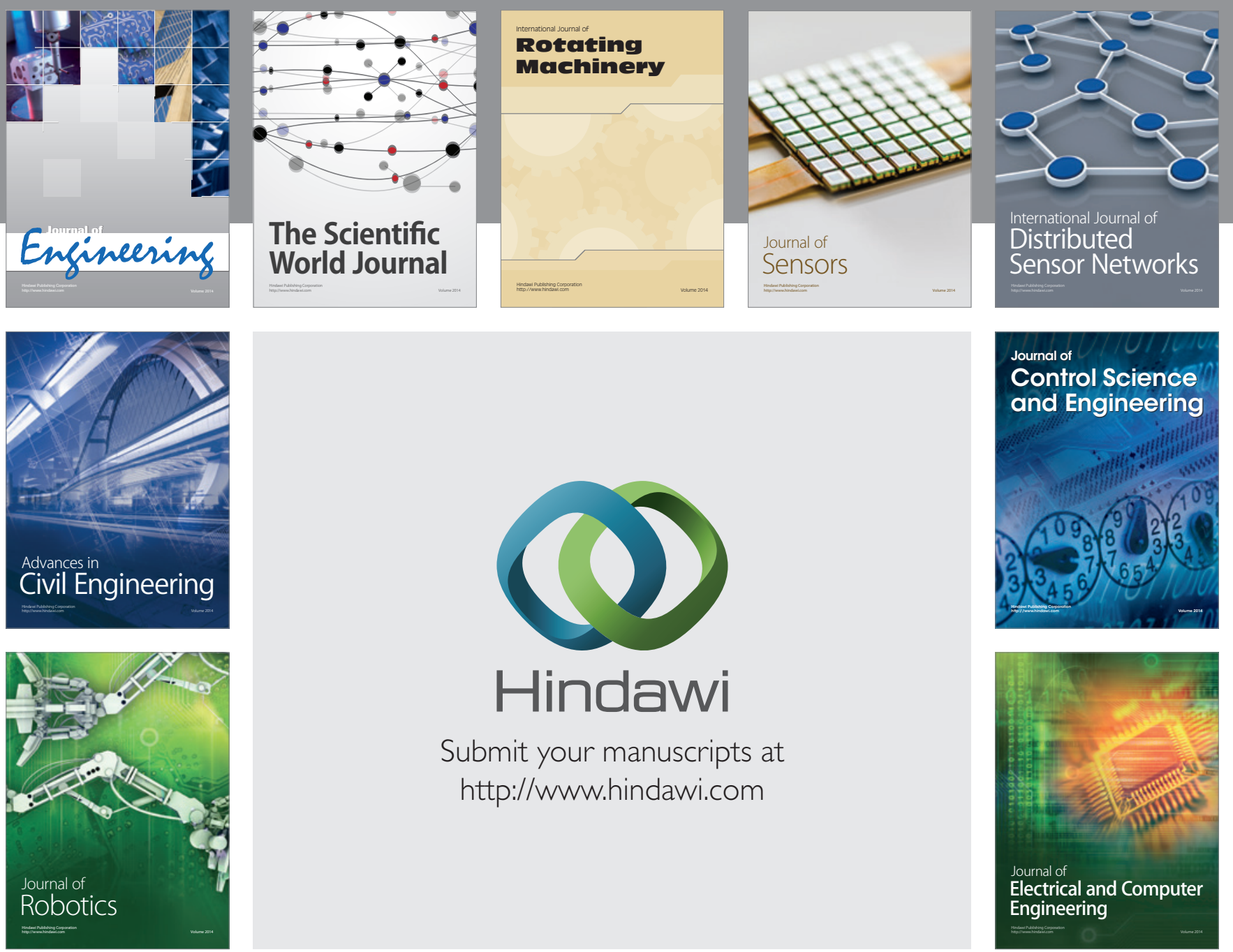

Submit your manuscripts at

http://www.hindawi.com
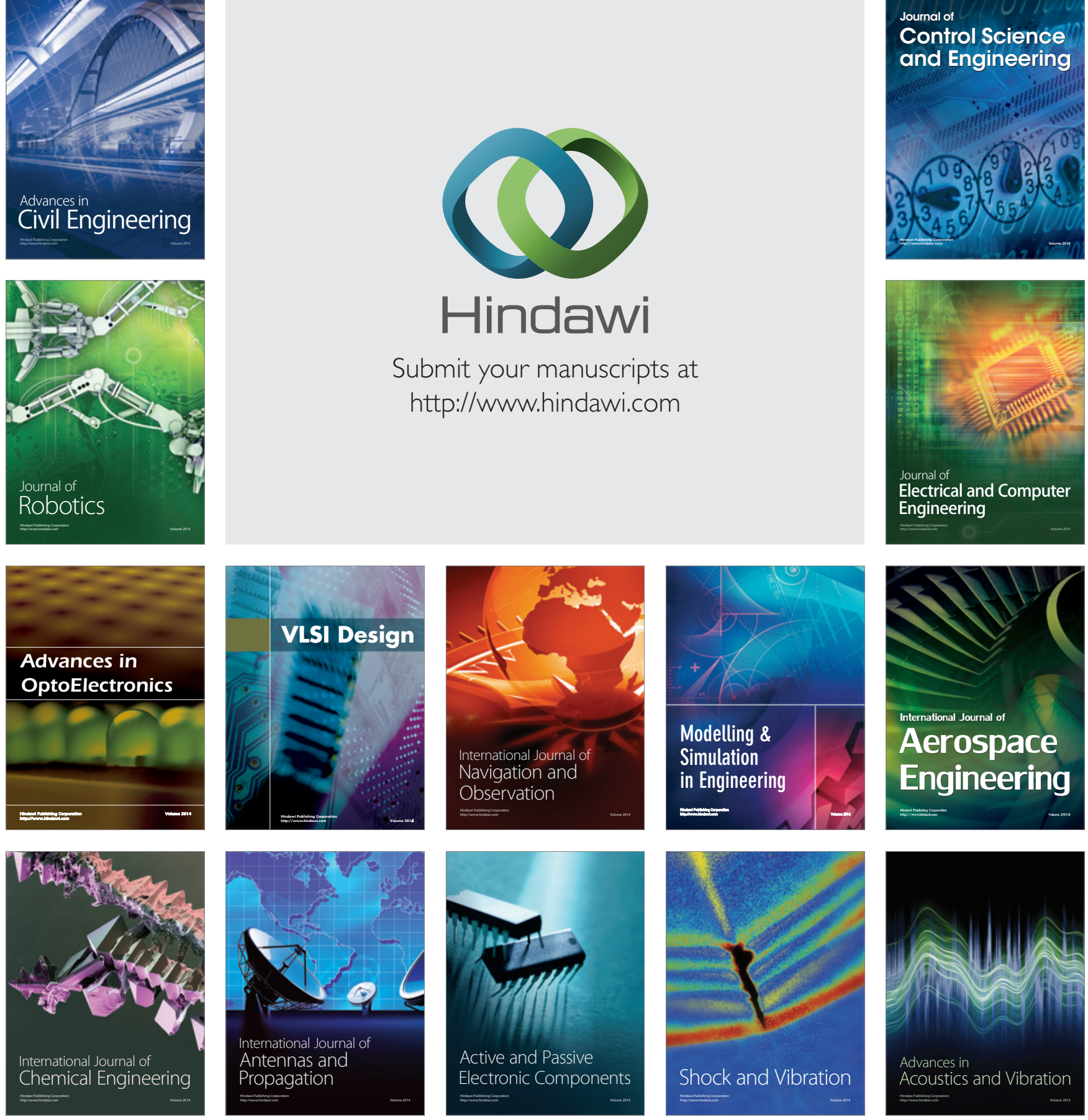